\title{
Contrast-Enhanced Cranial Computed Tomography in Magnetic Resonance Imaging Era
}

\author{
Fayaz A. Chishti ${ }^{a}$ Osama M. Al Saeed ${ }^{b}$ Hana Al-Khawari ${ }^{b}$ Mehraj Shaikh ${ }^{b}$ \\ aDepartment of Clinical Radiology, Al Amiri Hospital, Ministry of Public Health, bepartment of Radiology, \\ Faculty of Medicine, Kuwait University, Kuwait
}

\section{Key Words \\ Cranial computed tomography $\cdot$ Intravenous contrast}

\begin{abstract}
Objective: To evaluate the usefulness of intravenous contrast administration in cranial computed tomography (CT) in a general hospital with a magnetic resonance imaging (MRI) facility, and to establish a protocol to determine which patients would benefit most from using contrast-enhanced cranial CT. Subjects and Methods: Five hundred and forty-seven patients who underwent routine nonenhanced CT (NECT) and contrast-enhanced CT (CECT) of the brain between June 1997 and June 2001 were divided into three groups. Group A: 496 patients in whom CECT was done in spite of normal NECT; group B: 16 patients in whom CECT was considered necessary irrespective of NECT findings, and group C: 35 patients in whom NECT was abnormal and CECT was performed. Results: Contrast-enhanced cranial CT changed and/or confirmed the diagnosis in 1 of 496 in group A, 2 of 16 in group $B$, and 12 of 35 in group $C$, thereby indicating that CECT was useful in the diagnosis of groups $B$ and C. Conclusion: CECT is unlikely to be useful in patients with nor-
\end{abstract}

mal NECT in the appropriate clinical setting. A protocol is presented for the use of contrast media in cranial CT in a general hospital with an MRI facility. Using this protocol only $9.7 \%$ of patients for cranial CT would have needed CECT, resulting in considerable cost savings without affecting the quality of the service to the patient in a general hospital.

Copyright $\odot 2003$ S. Karger AG, Base

\section{Introduction}

Although magnetic resonance imaging (MRI) is the best investigational tool for cranial pathologies, only selected cranial cases undergo MRI due to limited access at many hospitals. Computerized tomography (CT) scanning of the cranium is therefore still performed in many hospitals $[1,2]$. Equally important is the fact that thirdgeneration CT scanners have good spatial resolution that enables maximum information to be obtained even from nonenhanced CT (NECT) of the cranium. Alterations in the blood-brain barrier due to any brain insult such as infection or neoplasm result in local enhancement of brain parenchyma after injection of iodinated contrast

\begin{tabular}{|c|c|c|}
\hline $\begin{array}{l}\text { KARGER } \\
\text { Fax }+41613061234\end{array}$ & $\begin{array}{l}\text { (c) } 2003 \text { S. Karger AG, Basel } \\
1011-7571 / 03 / 0124-0248 \$ 19.50 / 0\end{array}$ & $\begin{array}{l}\text { Dr. F.A. Chishti } \\
\text { PO Box } 8842 \\
22059 \text { Salmiya (Kuwait) }\end{array}$ \\
\hline $\begin{array}{l}\text { E-Mail karger@karger.ch } \\
\text { www.karger.com }\end{array}$ & $\begin{array}{l}\text { Accessible online at: } \\
\text { www. karger.com/mpp }\end{array}$ & $\begin{array}{l}\text { Tel. +965 5744259, Fax +965 } 5330473 \\
\text { E-Mail fachishti@hotmail.com }\end{array}$ \\
\hline
\end{tabular}


Table 1. Analysis of NECT and CECT findings

\begin{tabular}{|c|c|c|c|c|c|c|c|}
\hline \multirow[t]{2}{*}{ Clinical and CT findings } & \multirow[t]{2}{*}{ Patients } & \multicolumn{2}{|l|}{ NECT } & \multicolumn{2}{|l|}{ CECT } & \multirow{2}{*}{$\begin{array}{l}\text { Abnormality } \\
\text { in CECT } \\
\text { only }\end{array}$} & \multirow{2}{*}{$\begin{array}{l}\text { CECT } \\
\text { changed } \\
\text { diagnosis }\end{array}$} \\
\hline & & normal & abnormal & normal & abnormal & & \\
\hline $\begin{array}{l}\text { Group A } \\
\text { Patients who had clinically no focal signs and } \\
\text { NECT was normal }\end{array}$ & 496 & 496 & 0 & 495 & 1 & 1 & 1 \\
\hline $\begin{array}{l}\text { Group B } \\
\text { Patients in whom CECT was considered } \\
\text { necessary on the basis of clinical information, } \\
\text { irrespective of NECT being normal or abnormal }\end{array}$ & 16 & 10 & 6 & 4 & 12 & 2 & 2 \\
\hline $\begin{array}{l}\text { Group C } \\
\text { Patients who had clinically no focal signs and } \\
\text { NECT was abnormal }\end{array}$ & 35 & 0 & 35 & 5 & 30 & 0 & 12 \\
\hline Total number of patients & 547 & 506 & 41 & 504 & 43 & 3 & 15 \\
\hline
\end{tabular}

media (CM). The enhancement characteristics of various pathological states as described by Kramer et al. [3] and Huckman et al. [4] are well established. In the era of MRI, however, the usefulness of contrast-enhanced CT (CECT) when the initial finding of NECT is normal has not been well established $[2,5]$. We have therefore conducted a prospective study to assess the usefulness of CECT of the brain in a general hospital with limited access for cranial MRI. Based on our results and previous reports [6-8], a protocol to use of CM in cranial CT is suggested.

\section{Subjects and Methods}

Between June 1997 and June 2001, 547 adult out- and inpatients underwent both NECT and CECT of the cranium. Contrastenhanced CT was not performed in patients in whom intravenous $\mathrm{CM}$ was contraindicated or in patients who were suspected of having demyelinating disease, cranial nerve pathology, sella pathology, infection, metastasis or neoplasm because such patients are usually referred directly for MRI in our hospital. Nonionic low osmolar CM (iohexol $300 \mathrm{mg} / \mathrm{ml}$ ) was used. Postcontrast images were obtained immediately after a bolus injection of $50 \mathrm{ml}$ of CM. Slice thickness was $5 \mathrm{~mm}$ for the posterior fossa and $10 \mathrm{~mm}$ for supratentorial structures without an interslice gap.

The images obtained were analyzed independently by 2 consultant radiologists, each with more than 10 years experience. Initially the NECT studies were grouped as normal or abnormal. These images were then reviewed by the same radiologist along with the CECT images and regrouped as normal or abnormal. Discrepancies in the evaluation by the 2 radiologists of the same image were resolved by consensus after mutual review of the images. The abnormal images were reviewed together by the radiologists to determine whether or not the CECT changed the initial diagnosis of the patient.

For analysis, the findings were divided into three groups.

(1) Group A: normal NECT.

(2) Group B: irrespective of NECT findings, CECT was considered necessary on the basis of clinical presentation, including a history of leukemia, lymphoma, infectious symptomatology with neurological findings, phacomatosis or follow-up in known intracranial malignancy. In our hospital, generally such patients are referred directly for MRI, unless they are present after normal working hours or the MRI machine is out of order.

(3) Group C: abnormal NECT.

\section{Results}

A summary of the findings is given in table 1. In group A, of the 496 patients, none had abnormal finding on NECT but 1 was abnormal on CECT, thereby leading to a change in diagnosis. This was the case of a 68-year-old male with small meningioma (fig. 1). In group B, of the 16 patients, 10 were abnormal on NECT, which was confirmed by CECT. However, 2 more abnormal cases were noted on the CECT ( 2 patients with meningitis showing meningeal enhancement), thus leading to change in diagnosis. In group $\mathrm{C}$, of the 35 patients, 30 were abnormal and 5 equivocal on NECT. The 5 equivocal cases were confirmed normal on CECT and hence 30 were true positive abnormal cranial CT. The CECT changed the initial diagnosis in 7 abnormal cases on NECT. The CECT was particularly useful in differentiating atypical postischemic gliosis from malignancy in 4 patients and diagnosing vas- 

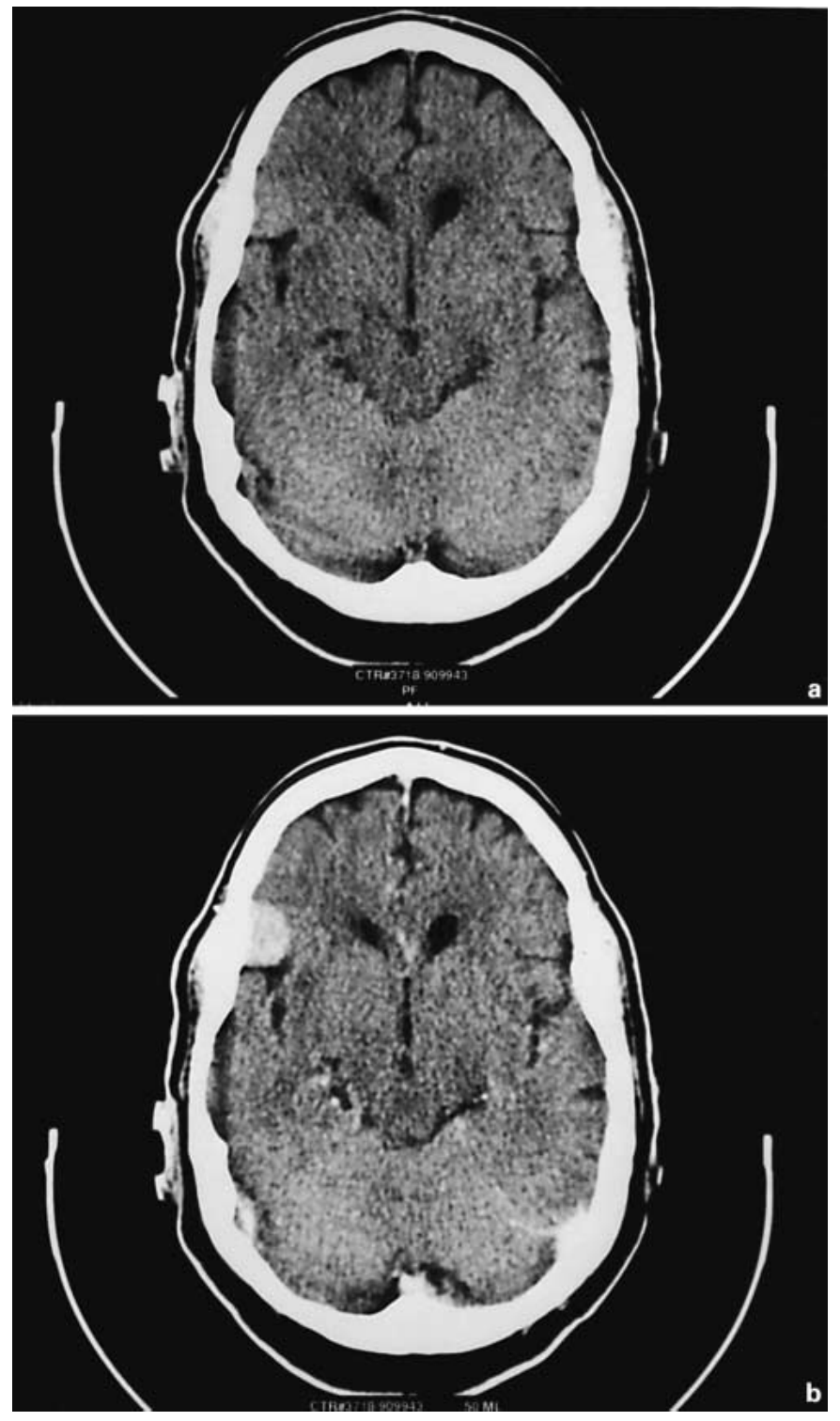

Fig. 1. a Nonenhanced CT. b Contrast-enhanced CT showing enhanced meningioma.

cular malformation in 3 patients. Thus in group C, CECT changed the diagnosis of 12 cases.

Overall, of the 547 patients, $43(7.8 \%)$ showed abnormal enhancement (table 1). In 3 cases (0.5\%), an abnormality was seen only on CECT. All abnormalities seen on NECT were visualized on CECT. The CECT changed the initial diagnosis in 15 cases out of $547(2.7 \%)$.

\section{Discussion}

Cranial CT constitutes 70\% of CT scans performed in our department. Performing CECT doubles the examination time and increases the radiation dose to the patient, cost of the examination, and patient morbidity [9].

In group A with 496 patients, the clinical information on the $\mathrm{CT}$ requests did not indicate any true localizing features, but the clinician did raise the possibility of intracranial space-occupying lesion, thereby making it difficult to decide whether or not to perform CECT based on the limited clinical information available at the time of scan. It is now clear that CECT did not add any further information to the patients with normal NECT except only in 1 case of small meningioma in a 65-year-old male patient (fig. 1). The patient was followed conservatively on the suggestion of neurosurgeon. In group B, in addition to confirming the abnormal cases of NECT, CECT showed 2 more abnormal cases that were normal on NECT and demonstrated meningeal enhancement consistent with clinical suspicion of meningitis. Equally important is the fact that CECT was considered helpful in all of the 16 patients of group B because it would not have been possible to conclude that the 6 cranial CT were indeed normal without administration of $\mathrm{CM}$. In group $\mathrm{C}$, of the 35 patients, CECT was useful in changing the diagnosis of 12 . By changing 5 cases from abnormal to normal, CECT was helpful in decreasing the number of false positive cases. In the other 7 cases, CECT was helpful in making differential diagnosis of atypical infarct or gliosis from malignancy and confirming the diagnosis of vascular malformation.

Hence the findings indicate that CECT was not necessary in group A but useful in groups B and C. Group B benefited because conditions such as lymphoma or metastasis may be small or localized along the ventricular and can only be properly visualised after contrast enhancement as previously reported [7]. In group C, CECT not only helped confirm the abnormality seen on NECT but also to make the final diagnosis, thereby decreasing either false positive or negative cases seen on NECT.

The exclusion criteria of not performing CECT in patients with contraindication to $\mathrm{CM}$ and direct referral for MRI did not constitute selection bias in the study, because this is precisely the basis of the study to attempt to determine the usefulness of CECT in an institution with limited access to an MRI facility. MRI is known to be more sensitive than $\mathrm{CT}$ in detecting brain lesions, particularly those less than $2 \mathrm{~cm}$ in diameter [10]. In patients with seizure disorders, sensitivity of CT is only $32 \%$ while that of MRI is $95 \%$ [11]. 
Based on the findings of this study and previous reports [9-11], we suggest the following guidelines for administration of intravenous contrast in cranial CT:

(1) Clinical history suggestive of trauma or vascular event: perform NECT only.

(2) Clinically no focal signs/symptoms: perform NECT, and after review by an experienced radiologist, if normal do not perform CECT, but further clinical review may be warranted to determine if MRI is necessary. If NECT is abnormal but not diagnostic, perform CECT. If NECT is abnormal and diagnostic, do not perform CECT. However, MRI may be performed for more anatomical details.

(3) Clinically suggestive of infection, neoplasm, demyelination, seizure disorder: do not perform $\mathrm{CT}$, rather refer the patient directly for MRI.

When the above guidelines are applied to this study, only 1 patient $(0.2 \%)$ in group A would have had a missed diagnosis and the 51 patients $(9.3 \%)$ in groups $\mathrm{B}$ and $\mathrm{C}$ would need CECT, thereby leading to considerable savings in cost without compromising the quality of service to the patients. Our result is less than that of previous reports $(23 \%)[6,11]$ possibly because their patients were not referred directly for MRI as was done in this study.

\section{Conclusion}

The findings indicate that CECT is not required in patients with normal NECT but is required in patients with equivocal and abnormal CECT. Patients with a history of leukemia, lymphoma, infections symptomatology with neurological findings, phacomatosis or follow-up in known intracranial malignancy should be referred directly for MRI.

\section{References}

1 Barrington AN, Lewtas NA: Indications for contrast medium enhancement in computed tomography of the brain. Clin Radiol 1977;28: 535-537.

2 Bernard MS, Hourihan MD, Adams H: Computed tomography of the brain: Does contrast enhancement really help? Clin Radiol 1991;44: 161-164.

3 Kramer RA, Janetos GP, Peristein G: An approach to contrast enhancement in computer tomography of the brain. Radiology 1975;116: 641-647.

4 Huckman MS: Clinical experience with intravenous infusion of iodinated contrast material as an adjunct to computed tomography. Surg Neurol 1975;4:297-318.
5 Moseley I: The use of contrast media as an adjunct to CT scanning; in Kvehler WJ (ed): Computer Assisted Tomography. Oxford, Excerpta Medica, 1977, pp 26-45.

6 Demaerel P, Buelens C, Wilms G, Baert AL: Cranial CT revisited: Do we really need contrast enhancement? Eur Radiol 1998;8:14471451.

7 Golfieri R, Cherryman GR, Olliff JF, Husband JE: Comparative evaluation of computerized tomography/magnetic resonance $(1.5 \mathrm{~T})$ in the detection of brain metastasis (in Italian). Radiol Med (Torino) 1991;82:27-34.
8 Bronen RA, Fulbright RK, Spencer DD, Spencer SS, Kim JH, Lange RC, Sutilla C: Refractory epilepsy: Comparison of MR Imaging, CT and histopathological findings in 117 patients. Radiology 1996;201:97-105.

9 Grainger R: What adverse reactions can be expected after administration of contrast media; in Dawson P, Clauss W (eds): Contrast Media in Practice. Springer, Berlin, 1993, pp 107-109.

10 Goldstein JD, Zeifer B, Chao C, Moser FG, Dickson DW, Hirschfeld AD, Davis L: CT appearance of primary CNS lymphoma in patients with acquired immunodeficiency syndrome. J Comput Assist Tomogr 1991;15:39.

11 Demaerel P, Wilms G, Baert AL: Is contrast enhancement in cranial computed tomography necessary? (letter). Lancet 1995;345:389. 